\title{
Image Retrieval of Earth-like Planets from Light Curves
}

\author{
Hajime Kawahara ${ }^{1}$ and Yuka Fujii ${ }^{2}$ \\ ${ }^{1}$ Department of Earth and Planetary Science, The University of Tokyo \\ Tokyo 113-0033, Japan \\ email: kawahara@eps.s.u-tokyo.ac.jp \\ ${ }^{2}$ Department of Physics, The University of Tokyo \\ Tokyo 113-0033, Japan
}

\begin{abstract}
Surface environment of habitable exoplanets will be important for astrobiologists on exoplanets in near future. Diverse surface environments on the Earth including continents, ocean, and meteorological condition (clouds and rains) serve as the backbone of biodiversity. One of the promising approaches to know the landscape of the terrestrial exoplanets is to use scattered light of the planet through direct imaging.

Since spin rotation and orbital revolution change illuminating area on planetary surface and cause time variation to disk-integrated brightness, light curves carry spatial information on the planetary surface. We propose an inversion technique of annual reflected light curves to sketch a two-dimensional albedo map of exoplanets, named the spin-orbit tomography (SOT). Applying the SOT to realistic simulations of the reflected light of an Earth-twin, we demonstrate how the SOT works. The mean cloud and continental distributions can be roughly obtained with single band photometry and difference of two-bands photometry, respectively. The SOT retrieves the planetary image without actually resolving the planet, which can be used to know the habitat of the exoplanets in near future.
\end{abstract}

Keywords. Earth-like planets, Mapping, Scattered lights

\section{How can we take a spatially resolved image of an Earth-like planet?}

In near future, it will be important for astrobiology to understand the environment of exo-planetary surface, in other words, the habitat of the exoplanet. Indeed, diverse surface environments on Earth including continents, ocean, and meteorological condition serve as the backbone of biodiversity. One of the promising approaches to know the landscape of the terrestrial exoplanets is to identify surface components using the scattered light of the planets through direct imaging observations.

However, even nearby Earth-like planets are so small. Using a large number of space telescopes (for instance, $3 \mathrm{~m}$ aperture $\times 150$ ), as proposed by Labeyrie (1999), is one possibility to resolve the nearby exoplanets. Even if one cannot resolve the planet directly, the reflected light curve from the Earth-like planets itself contains information on the habitat on the planetary surface, such as clouds, soil, ocean, and vegetation since they have different reflectivity (Ford et al. (2001), Cowan et al. (2009), Oakley \& Cash (2009), Fujii et al. (2010), Kawahara \& Fujii (2010), Fujii et al. (2011), Kawahara \& Fujii (2011), Cowan et al. (2011), Fujii \& Kawahara (2012), and references therein). Our goal is to develop an inversion technique of annual reflected light curves to sketch a two-dimensional albedo map of the exoplanets, which will enable us to indirectly resolve the planet with a single space telescope. 


\section{Spin-Orbit Tomography: Inversion Technique of the Planetary Surface from Disk-integrated Light Curve}

The reflected light from a planet depends on albedo of the illuminating region of the planetary surface, which changes according to planet's rotation and orbital revolution. Using this fact, we developed the inversion method to retrieve the reflectivity map from the time series of reflected light curve. Since we use diurnal and annual variation of the reflected light, we call our method Spin-Orbit Tomography (SOT; Kawahara \& Fujii (2010), Kawahara \& Fujii (2011), Fujii \& Kawahara (2012)). The SOT of the mock Earth light curve primary provides cloud distribution since clouds dominate reflected light as shown in Figure 1.

Applying the SOT to the reflectivity difference of 2 bands, one can sketch the continental distribution due to the wavelength dependence of albedo of soil or vegetations. Even for low-oblique planet like Earth, the SOT can reconstruct the continental distribution. For instance, half year observation of Earth twin at $10 \mathrm{pc}$ with a 4-13 m space telescope will give a high quality map of the surface shown in Figure 2.

\section{Measurement of Exoplanetary Spin Vector}

The spin of an exoplanet is one of important parameters to determine the habitability and its measurement has the potential to constrain the formation theory (e.g. Kokubo \& Ida (2007)). The SOT provides a means to measure the spin vector for direct imaging (see Kawahara \& Fujii (2010), Kawahara \& Fujii (2011), Fujii \& Kawahara (2012)). We have also proposed a new method with planetary radial velocity (PRV) to constrain the spin vector (Kawahara (2012)). The PRV in dayside spectra was actually measured for
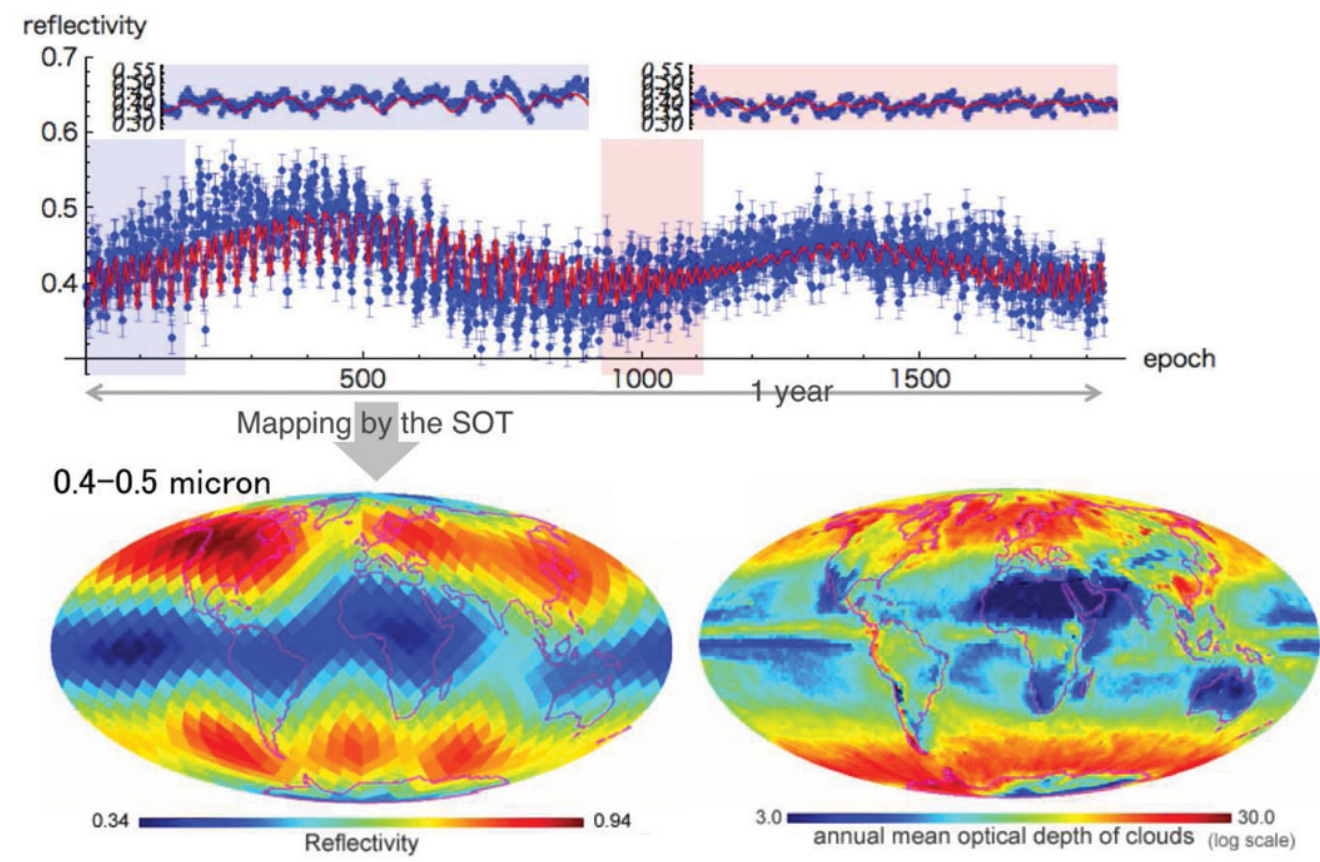

Figure 1. A simulated one-year light curve of Earth in the 0.4-0.5 $\mu \mathrm{m}$ band (upper panel; this band is mainly dominated by reflection of clouds) and its retrieved map (bottom left). The bottom right panel shows annual mean distribution of clouds. See Kawahara \& Fujii (2011). 

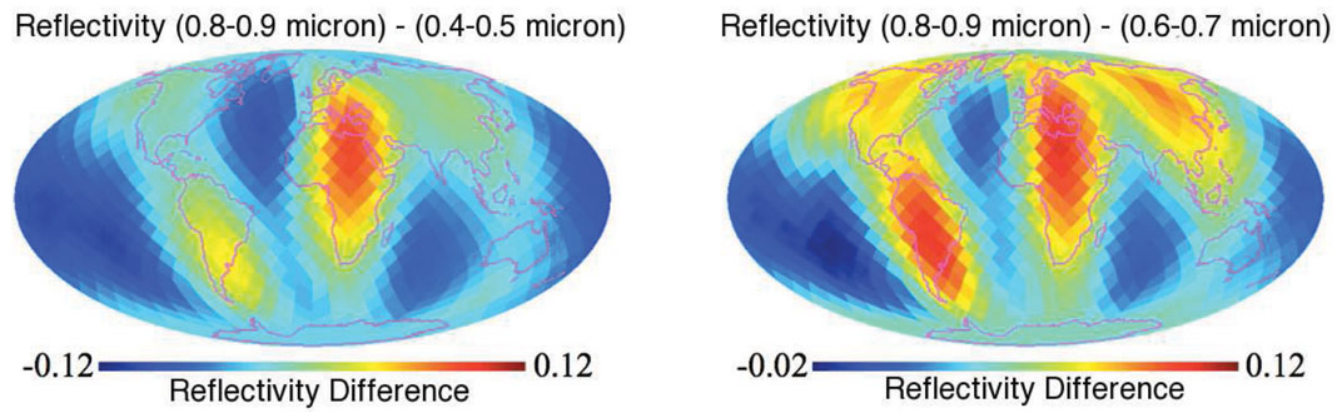

Figure 2. The retrieved maps from the colors of simulated scattered light. We use the reflectivity difference between $[0.8-0.9]-[0.5-0.4] \mu \mathrm{m}$ (left) and [0.8-0.9] - [0.7-0.6] $\mu \mathrm{m}$ (right). Since the retrieved map in the right panel uses the bands across the red edge, the signal at Latin America (Amazon) in the right panel is stronger than that in the left panel. See Fujii \& Kawahara (2012).

$\tau$ Boötis b, recently (Brogi et al. (2012), Rodler et al. (2012)). Though this method only provide a sky projected angle between the spin axis and orbital axis, it can constrain the planetary obliquity. Thus the information on the planets's spin will be available via the PRV for close-in giant planets or super-Earths, and via the SOT for Earth-size planets observed by direct imaging.

\section{References}

Brogi, M., Snellen, I. A. G., de Kok, R. J., Albrecht, S., Birkby, J., \& de Mooij, E. J. W. 2012, Nature, 486, 502

Cowan, N. B., et al. 2009, The Astrophysical Journal, 700, 915

Cowan, N. B., et al. 2011, The Astrophysical Journal, 731, 76

Ford, E. B., Seager, S., \& Turner, E. L. 2001, Nature, 412, 885

Fujii, Y. \& Kawahara, H. 2012, The Astrophysical Journal, 755, 101

Fujii, Y., Kawahara, H., Suto, Y., Fukuda, S., Nakajima, T., Livengood, T. A., \& Turner, E. L. 2011, The Astrophysical Journal, 738, 184

Fujii, Y., Kawahara, H., Suto, Y., Taruya, A., Fukuda, S., Nakajima, T., \& Turner, E. L. 2010, The Astrophysical Journal, 715, 866

Kawahara, H., 2012, The Astrophysical Journal Letters, 760, L13

Kawahara, H. \& Fujii, Y. 2010, The Astrophysical Journal, 720, 1333

Kawahara, H. \& Fujii, Y. 2011, The Astrophysical Journal Letters, 739, L62

Kokubo, E. \& Ida, S. 2007, The Astrophysical Journal, 671, 2082

Labeyrie, A. 1999, Astronomical Society of the Pacific Conference Series, 194, 350

Oakley, P. H. H. \& Cash, W. 2009, The Astrophysical Journal Letters 700, 1428

Rodler, F., Lopez-Morales, M., \& Ribas, I. 2012, The Astrophysical Journal Letters 753, L25 